\title{
Measurement of Pressures Generated in Root Canal During Er:YAG Laser-Activated Irrigation
}

\author{
Matija Jezeršek, PhD, ${ }^{1}$ Nejc Lukač, $\mathrm{PhD},{ }^{1}$ Matjaž Lukač, $\mathrm{PhD},{ }^{2}$ Ana Tenyi, $\mathrm{DMD},{ }^{3}$ \\ Giovanni Olivi, DMD, ${ }^{4}$ and Aleš Fidler, DMD, $\mathrm{PhD}^{3,5}$
}

\begin{abstract}
Objective: To measure distribution of pressures along the depth of the root canal during erbium-doped yttrium aluminum garnet (Er:YAG) laser-activated irrigation (LAI) with different modalities and fiber tip (FT) geometries.

Background: A new LAI modality based on the delivery of synchronized pairs of Er:YAG laser pulses to generate enhanced irrigant streaming and shock wave emission was recently introduced. However, the influence of FT geometry on efficacy and comparison with single pulse modality is not yet presented.

Methods: Pressures within a simulated root canal were simultaneously measured at 5 depths during LAI. Seven FT geometries (conical and cylindrical) and two modalities [Super Short Pulse (SSP) and dual pulse AutoSWEEPS] were compared.

Results: Under the same conditions, average pressures using SSP at $20 \mathrm{~mJ}$ of laser energy ranged from $111 \mathrm{~Pa}$ for a conical $600 \mu \mathrm{m}$ FT to $225 \mathrm{~Pa}$ for a flat $400 \mu \mathrm{m}$ FT. The measured pressures for the SSP and the AutoSWEEPS at $20 \mathrm{~mJ}$ laser energy were 223 and $308 \mathrm{~Pa}$ at the most coronal level and 119 and $126 \mathrm{~Pa}$ at the apical constriction, respectively. Measured pressures and irrigant penetration depths at different root canal levels were found to be linearly correlated $\left(R^{2}=0.82 ; p<0.01\right)$.

Conclusions: The generated pressures get progressively reduced from the coronal toward the apical third of the root canal. A strong dependence on the FT design and laser modality was observed. Within the limitations of the study, the AutoSWEEPS modality is more effective than standard SSP in generating pressures within the root canal, without increasing the risk of extrusion.
\end{abstract}

Keywords: cavitation, laser-activated irrigation, pressure, root canal

\section{Introduction}

$\mathbf{E}$ NDODONTIC THERAPY CONSISTS of the elimination of pathogenic substances from the root canal system. ${ }^{1}$ Due to the complexity of the three-dimensional (3D) anatomy of the root canal system, the standard mechanical instrumentation leaves a significant portion $(20-60 \%)$ of the root canal wall un-instrumented. ${ }^{2}$ Further, instrumentation creates a smear layer and accumulation of debris in surface irregularities. $^{3}$ For this reason, the irrigation phase of the therapy is necessary to eradicate the potential existing pathogens, and to remove the smear layer and debris produced during the instrumentation procedures.
Several different techniques have been introduced to improve the efficacy of the standard syringe root canal irrigation. ${ }^{4-8}$ More recently, laser-activated irrigation (LAI) was proposed due to its capability to generate micro-cavitations within the irrigant-filled root canal system. ${ }^{9-12}$ Typically, LAI is achieved through the high absorption of an erbiumdoped yttrium aluminum garnet (Er:YAG) laser pulse in the irrigant, which initiates a rapid formation of a vapor bubble at the fiber tip (FT) while it is immersed in the irrigant. ${ }^{13,14}$ This phenomenon induces a turbulent fluid movement within the whole canal volume, and it was reported to improve the efficacy of chemo-mechanical debridement. ${ }^{12,15-18}$ An example of LAI is photon-induced photoacoustic streaming (PIPS)

\footnotetext{
${ }^{1}$ Faculty of Mechanical Engineering, Laboratory for Laser Techniques, University of Ljubljana, Ljubljana, Slovenia.

${ }^{2}$ Department of Complex Matter, Jozef Stefan Institute, Ljubljana, Slovenia.

${ }^{3}$ Medical Faculty, Department of Endodontics and Restorative Dentistry, University of Ljubljana, Ljubljana, Slovenia.

${ }^{4}$ Master Laser Dentistry, Catholic University of Sacred Heart of Rome, Roma, Italy.

${ }^{5}$ Department of Endodontics, University Clinical Centre, Ljubljana, Slovenia.
} 
a

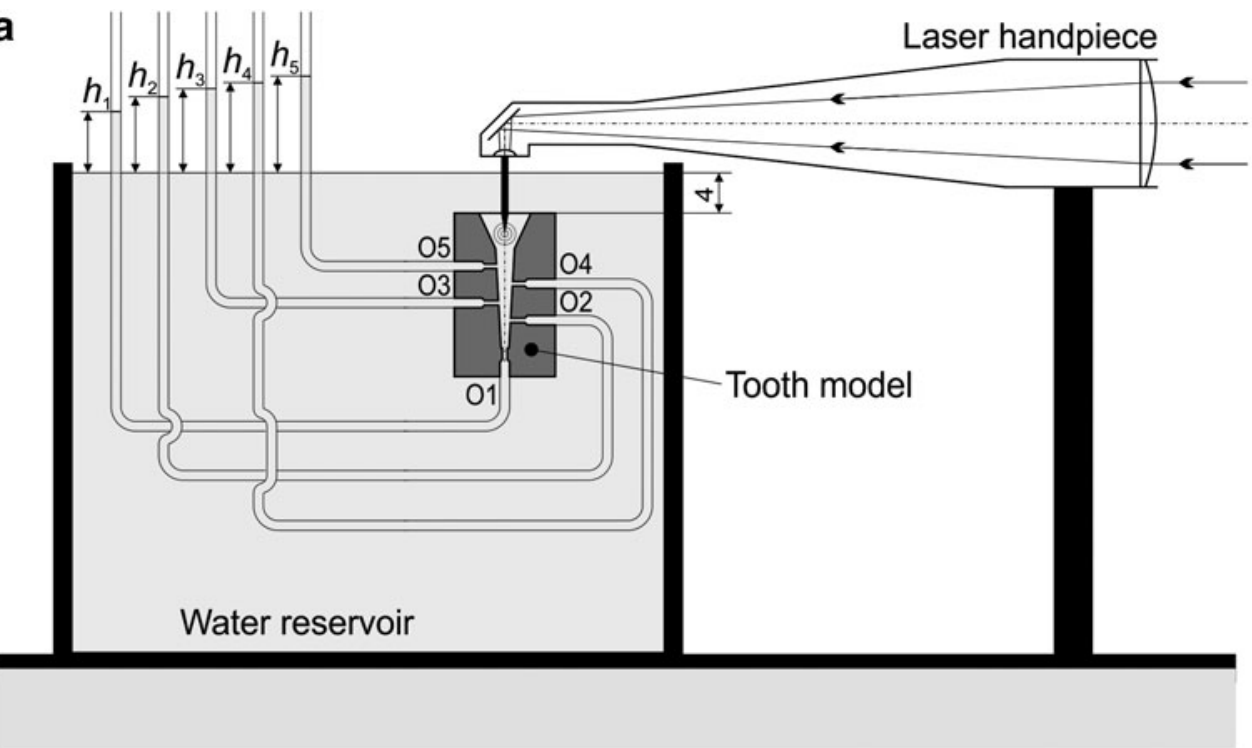

b

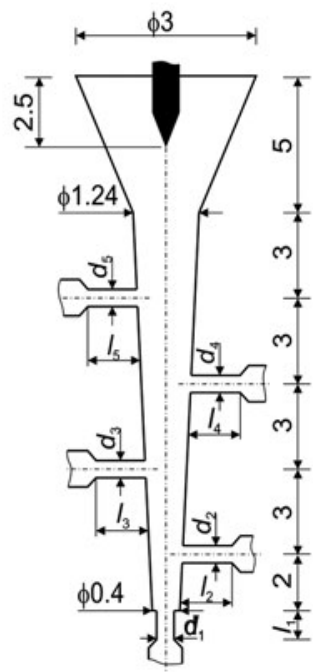

FIG. 1. Experimental system for measuring pressures at different locations within the model root canal (a) and geometry of the model root canal (b). The lateral constrictions $(i=2 \ldots 5)$ had approximate diameters $d_{i}=0.45 \mathrm{~mm}$ and lengths $l_{i}=3.5 \mathrm{~mm}$, whereas the vertical (apical) constriction's approximate dimensions were $d_{1}=0.2 \mathrm{~mm}$ and $l_{1}=1 \mathrm{~mm}$. The location of the laser FT in the access cavity is represented by the tapered black tip. FT, fiber tip.

performed by single Super Short Pulse (SSP) with a duration of $50 \mu \mathrm{s}$ Er:YAG laser pulses. ${ }^{14-16}$ The latest LAI development is the SWEEPS (Shock Wave Enhanced Emission Photoacoustic Streaming) laser modality, which is based on the delivery of pairs of Er:YAG laser pulses, timed in such a manner to generate enhanced irrigant streaming and shock wave emission. $^{12,18,19-21}$

Various experimental approaches have been used to study the effects of LAI. ${ }^{13,15,21,22}$ In one set of experiments, ${ }^{18,23}$ penetration depths of various dyes into dentinal tubules were studied as a surrogate measure to investigate the ability of different irrigation solutions to penetrate the root canal walls. With the laser tip in the pulp chamber, the penetration depth of the irrigants decreases from the coronal to the apical third of the canal. ${ }^{18,23}$

The efficacy and safety of endodontic irrigation have also been evaluated by measuring the resulting fluid pressure during irrigation. ${ }^{24-26}$ There are, however, not many published studies of direct measurements of fluid pressures during LAI. In a study by Yao et al., ${ }^{27}$ the pressure generated within the root canal during LAI was measured at one location, outside the apical foramen.

In this study, simultaneous measurements of fluid pressures were made for the first time at different regions located from coronal to apical level of a root canal model. The measured pressures were used to evaluate the influence of laser modality and FT geometry on the efficacy and safety of LAI irrigation. The results were further applied to compare the measured pressures with published dentinal tubule penetration depths for two Er:YAG LAIs protocols. ${ }^{18}$

\section{Materials and Methods}

\section{Experimental set-up}

The experimental system for the measurement of pressures at different depths within a model root canal is shown in Fig. 1. The simulated root canal model (DRSK Group AB, Hässleholm, Sweden) has a conically shaped access cavity with $5 \mathrm{~mm}$ length and $3 \mathrm{~mm}$ diameter. The canal had a length of $14 \mathrm{~mm}$, a diameter of 0.40 at the tip, and a 0.06 taper over the first apical $3 \mathrm{~mm}$. The canal was submerged $4 \mathrm{~mm}$ deep under the water level of a large water-filled reservoir to ensure the constant replenishment of irrigant, and to provide a stable fluid pressure within the root canal in the absence of LAI.

LAI was performed by using an Er:YAG laser (LightWalker, Fotona d.o.o., Slovenia) equipped with a dental handpiece (H14) optically coupled with an interchangeable FT. The handpiece air/water spray was turned off during all experiments. Two laser modes were used in the study: (1) SSP mode with a laser pulse duration of $50 \mu$ s (Table 1), and (2) AutoSsWEEPS mode, based on $25-\mu$ s pulse pairs with

Table 1. Laser Parameters for the Evaluation OF THE INFLUENCE OF FibER TIP GEOMETRY

\begin{tabular}{|c|c|c|c|}
\hline Group & $F T$ & Laser device & $\begin{array}{c}\text { Laser parameters } \\
\text { (same for all } \\
\text { groups) }\end{array}$ \\
\hline $\mathrm{a}$ & Flat Sweeps400 & LightWalker & Wavelength: \\
\hline $\mathrm{b}$ & Flat Sweeps 400 & SkyPulse & 2940 nm; Pulse \\
\hline $\mathrm{c}$ & Radial Sweeps 400 & LightWalker & energy: $20 \mathrm{~mJ}$ \\
\hline $\mathrm{d}$ & Radial Sweeps 400 & SkyPulse & $\begin{array}{l}\text { Repetition rate: } \\
20 \mathrm{~Hz} \text {; Pulse } \\
\text { duration: } 50 \mu \mathrm{s} \\
\text { (SSP } \\
\text { modality); } \\
\text { Duration of } \\
\text { irrigation: } \\
80 \mathrm{sec}\end{array}$ \\
\hline
\end{tabular}

FT, fiber tip; SSP, Super Short Pulse. 
Table 2. Laser Parameters for the Evaluation of the Influence of Laser Modality

\begin{tabular}{|c|c|c|c|c|}
\hline Group & $F T$ & Pulse duration & Pulse energy $(\mathrm{mJ})$ & $\begin{array}{c}\text { Laser parameters } \\
\text { (same for all groups) }\end{array}$ \\
\hline a & Radial Sweeps600 & Single pulse $50 \mu \mathrm{s}$ (SSP modality) & 20 & Laser: LightWalker; \\
\hline $\mathrm{b}$ & Flat Sweeps400 & Two $25 \mu$ s micropulses separated & 10 & Wavelength: $2940 \mathrm{~nm}$; \\
\hline $\mathrm{c}$ & Flat Sweeps400 & $\begin{array}{l}\text { by continuously varied delay } \\
\text { between } 250 \text { and } 650 \mu \text { s with } \\
10-\mu \text { s steps (AutoSWEEPS } \\
\text { modality) }\end{array}$ & 20 & $\begin{array}{l}\text { Repetition rate: } 20 \mathrm{~Hz} \\
\text { Duration of irrigation: } 80 \mathrm{sec}\end{array}$ \\
\hline
\end{tabular}

repetitive sweeping of the temporal separation between the pulses from 250 to $650 \mu$ s (Table 2). ${ }^{28}$

The following FTs (manufactured by Fotona) were used in the study:

(1) Conical tips (Fig. 2a); conically shaped FTs with 400 and $600 \mu \mathrm{m}$ diameter at the exit end (Conical Sapphire400 and Conical Sapphire600)

(2) Flat tips (Fig. 2b); cylindrically shaped flat-ended FTs with 400, 500, and $600 \mu \mathrm{m}$ diameters (Flat Sweeps400, Flat Sweeps500, and FlatSweeps600)

(3) Radial tips (Fig. 2c); cylindrically shaped FTs with a tapered exit end, and 400 and $600 \mu \mathrm{m}$ diameters (Radial Sweeps400 and Radial Sweeps600)

According to the PIPS protocol, the laser FT's ending was positioned $2.5 \mathrm{~mm}$ deep into the access cavity (Fig. 1b), using an XYZ micrometer positioning stage. ${ }^{14}$ The FT position relative to the canal was monitored with a digital camera (Chameleon3, 1.3 MP, PointGrey, Richmond, Canada) with the optical resolution of $0.1 \mathrm{~mm}$.

\section{Pressure measurements}

The simulated root canal model had five pressure measuring regions $(\mathrm{O} 1 \ldots \mathrm{O} 5)$ located at different depths of the root canal (Fig. 1a), consisting of five tubular constrictions with the dimensions as shown in Fig. 1b. The constrictions were connected to tubes with an internal diameter of $1.6 \mathrm{~mm}$.

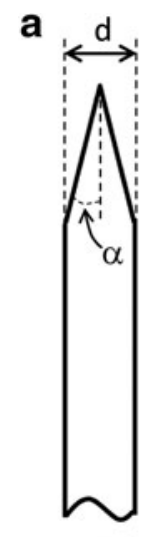

$\alpha=17 \mathrm{deg}$

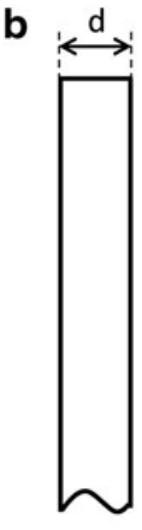

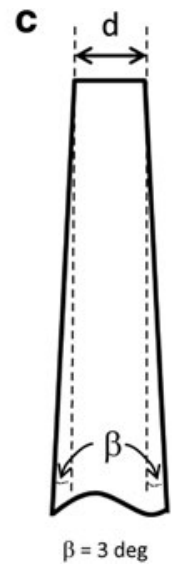

$\beta=3 \operatorname{deg}$
FIG. 2. FTs, used in the study. (a) Conical tips with 400 and $600 \mu \mathrm{m}$ diameter at the exit end. (b) Flat tipscylindrically shaped flat-ended with 400,500 , and $600-\mu \mathrm{m}$ diameters. (c) Radial tips-cylindrically shaped FTs with tapered exit end, and 400 and $600-\mu \mathrm{m}$ diameters.
The tubes ended with a vertical section extending $10 \mathrm{~cm}$ above the water level of the large reservoir (Fig. 1a).

The measuring principle is based on measurement of stationary water column heights inside each of the five tubes. A second digital camera (Chameleon3, 1.3 MP, PointGrey, Richmond, Canada) was used for measuring the water level heights $\left(h_{\mathrm{i}}\right)$ simultaneously for all five water columns, with a resolution of $0.25 \mathrm{~mm}$ and a frequency of 10 measurements per second. A custom computer program using graphical programming software (LabVIEW 2016, National Instruments, Austin) was developed for real-time detection of water levels from the acquired video data. The program detected brightness decrease within each tube, calculated the height difference between water levels within the tubes and the large reservoir, and stored the measured values, $h_{\mathrm{i}}$.

During simulated irrigation conditions, laser pulses were delivered through the FT into the access cavity at a constant repetition rate of $f=20 \mathrm{~Hz}$. When laser radiation was turned on, the fluid columns $h_{i}$ started to rise as a result of the increased pressure within the root canal, until they reached their stationary heights, $h_{o i}$ (Fig. 3). At that stage, the equilibrium is reached between upward volumetric fluid flow rate $Q_{u i}$ (in $\mathrm{mm}^{3} / \mathrm{sec}$ ) and downward fluid flow rate $Q_{d i}$ caused by the pressure difference in the connecting vessel due to the increased height of the water columns relative to the reservoir's water surface. Because of this pressure difference, the height of the water columns started to decrease immediately after laser radiation was turned off, at the height-dependent decrease rate of $v_{i}=\mathrm{d} h_{i} / \mathrm{d} t\left(h_{i}\right)$ (in $\mathrm{mm} / \mathrm{sec}$ ).

For column heights below $20 \mathrm{~mm}$, as was the case during our measurements, the height decrease rates $\left(v_{i}\right)$ in the absence of laser radiation were found to be approximately linearly dependent on the column height for all columns,

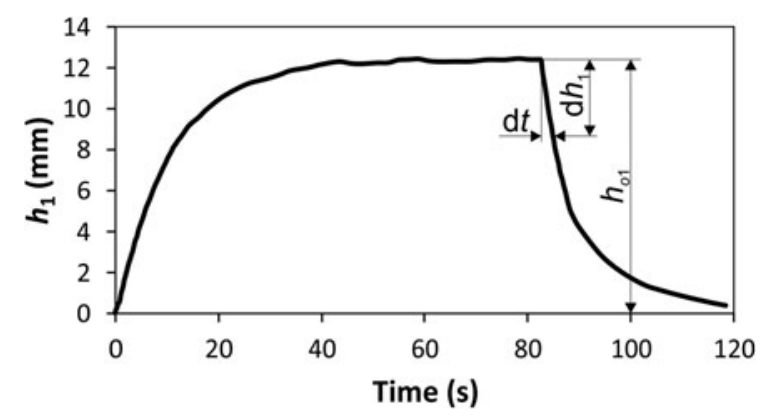

FIG. 3. Temporal evolution of the water column's height during and after laser radiation. The shown example is for the opening $\mathrm{O} 1$, with the duration of radiation of $80 \mathrm{sec}$. 
$v_{i}\left(h_{i}\right)=-k_{i} \times h_{i}$. This allowed the downward and upward average fluid flows at the equilibrium to be calculated from:

$$
Q_{u i}=Q_{d i}=k_{i} \times h_{0 i}
$$

The pressure differences $\left(P_{\mathrm{i}}\right)$ between the two ends of the narrow constrictions were calculated by using the HagenPoiseuille equation defining the pressure difference in an incompressible and Newtonian fluid that is required to result in a laminar flow rate $Q$ through a cylindrical pipe $\mathrm{p}^{29,30}$ :

$$
P_{i}=\mu \cdot Z_{i} \cdot Q_{u i}
$$

Here, $\mu$ is the fluid's viscosity $\left(\mu_{\text {water }}=8.90 \times 10^{-4} \mathrm{~Pa} \cdot \mathrm{s}\right.$ at $\left.25^{\circ} \mathrm{C}\right)$, and $Z_{i}=8 l_{i} /\left(\pi \cdot r_{i}^{4}\right)$ is the constriction's flow resistance.

In the presented results, each data point represents an average of three measurements of the equilibrium water level height for each tested experimental condition.

\section{Evaluation of the influence of FT geometry}

In the first part of the study, the influence of the FT geometry on the pressure generation with the SSP mode was evaluated. Average generated pressure for different FT types was calculated by using $P_{\text {ave }}=\left(P_{1}+P_{2}+P_{3}+P_{4}+P_{5}\right) / 5$.

Pressure distributions within the simulated root canal were measured in more detail for the SSP modality and two FT types (Flat Sweeps400 and Radial Sweeps400), which were found to be most effective for pressure generation. To exclude any dependence of the results on the type of laser beam delivery, an additional Er:YAG laser device (SkyPulse, Fotona d.o.o, Ljubljana, Slovenia) where the laser source is positioned at the entrance to the laser handpiece was used in this part of the study (groups b and c), in addition to LightWalker (groups a and d), equipped with an articulated arm beam delivery (Table 1). The same laser handpiece $\mathrm{H} 14$ was used with both laser devices.

\section{Evaluation of the influence of laser modality}

In the second part of the study, the Er:YAG laser was used in different modalities: (a) Standard SSP protocol using Radial Sweeps600 FT with the pulse energy $E_{L}=20 \mathrm{~mJ}^{16,18}$, (b) AutoSWEEPS mode using Flat Sweeps400 FT with $E_{L}=10$ $\mathrm{mJ}$, and (c) AutoSWEEPS mode using Flat Sweeps400 FT with $E_{L}=20 \mathrm{~mJ}$ (Table 2).

\section{Data analysis}

The Pearson correlation coefficient $\left(R^{2}\right)$ was calculated to assess the relationship between pressures measured in this study and published dentinal tubule penetration depths at apical, middle, and coronal root canal level. Evaluation of measured pressures at different laser modalities and canal levels was performed by the independent-samples $t$-test. A $p$-value of 0.05 was considered statistically significant. Statistical analysis was performed by software package (Stata, version 15, StataCorp LLC, College Station).

\section{Results}

Comparison of measured average pressures $P_{\text {ave }}$ for the single pulse SSP mode and different FTs (Fig. 4) shows both $400-\mu \mathrm{m}$ diameter FTs, (Flat Sweeps400 and Radial

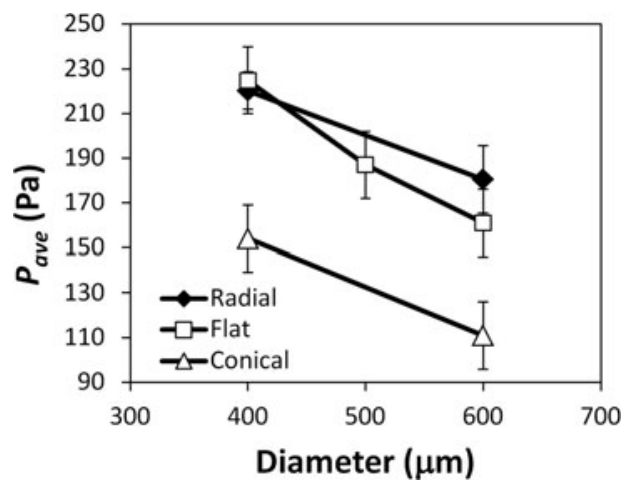

FIG. 4. Average pressure within the root canal for different FT geometries and diameters, and the same single pulse energy of $E_{L}=20 \mathrm{~mJ}$ in SSP mode. SSP, Super Short Pulse.

Sweeps400), to generate highest pressures within the root canal. In general, pressures within the root canal were observed to decrease with increasing fiber diameter, with the lowest pressure measured for the conically shaped FTs (Conical Sapphire400 and Conical Sapphire600).

The detailed pressure distributions within the root canal, as measured with two Er:YAG laser devices (LightWalker and SkyPulse) in the SSP mode, using the two most effective FTs from Fig. 4 (Flat Sweeps400 and Radial Sweeps400) are presented in Fig. 5. The irrigant pressures were observed to decrease from coronal to apical level. At each level, similar pressure values were found for the used flat and tapered FTs (Flat Sweeps400 and Radial Sweeps400) or Er:YAG laser beam deliveries (LightWalker and SkyPulse).

Figure 6 shows measured pressure distributions within the root canal as generated during the standard single pulse SSP protocol with $E_{L}=20 \mathrm{~mJ}^{16,18}$ using Radial Sweeps600 FT, and during two dual pulse AutoSWEEPS LAI protocols with single pulse energy $E_{L}=10$ and $20 \mathrm{~mJ}$ using Flat Sweeps 400 FT. In comparison to the single pulse SSP irrigation, the

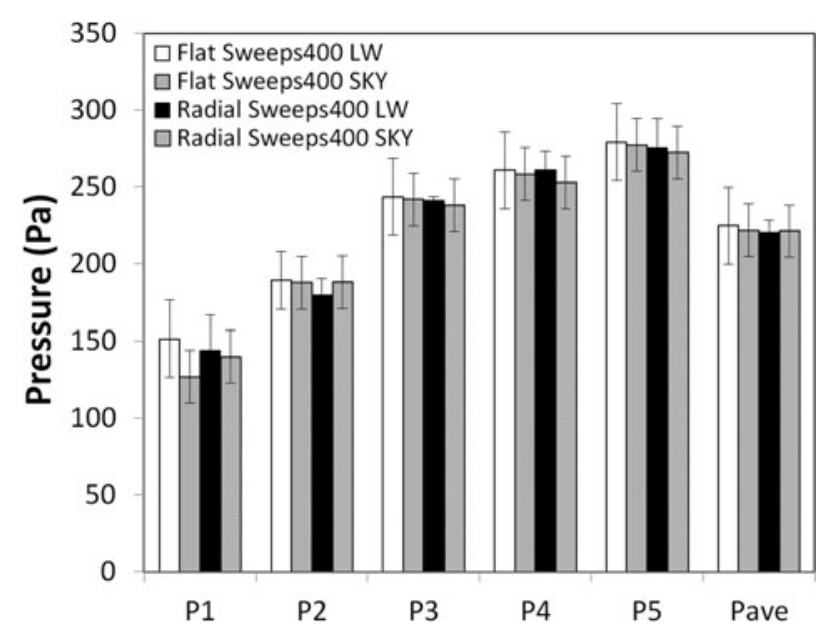

FIG. 5. Measured pressures at different locations within the root canal for the Flat and Radial Sweeps400 FT, with SSP mode at $E_{L}=20 \mathrm{~mJ}$, delivered by LightWalker (LW) or SkyPulse (SKY) Er:YAG laser device. Er:YAG, erbiumdoped yttrium aluminum garnet. 


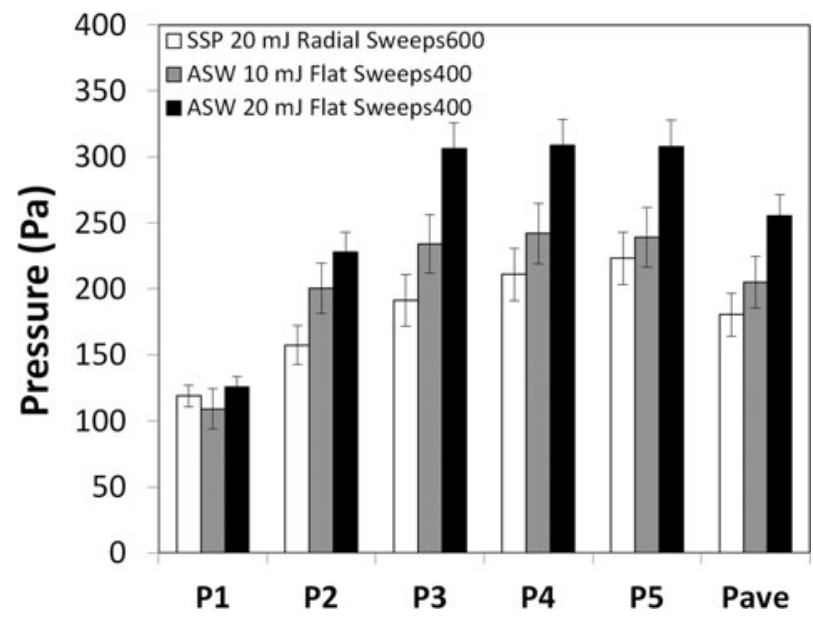

FIG. 6. Measured pressures within the root canal for the SSP and two different AutoSWEEPS protocols: SSP mode as used in Ref. ${ }^{18,23}$ with Radial Sweeps600 FT and $E_{L}=$ $20 \mathrm{~mJ}$; AutoSWEEPS mode with Flat Sweeps400 FT and $E_{L}=10 \mathrm{~mJ}$; and AutoSWEEPS mode with Flat Sweeps 400 FT and $E_{L}=20 \mathrm{~mJ}$.

tested dual pulse AutoSWEEPS irrigation protocols resulted in higher pressures in the middle and coronal areas, with lower or comparable pressures in the apex. A significant difference $(p<0.01)$ was observed between the average pressures as obtained with the SSP and AutoSWEEPS $10 \mathrm{~mJ}$ protocol, and with the AutoSWEEPS $10 \mathrm{~mJ}$ and AutoSWEEPS $20 \mathrm{~mJ}$ protocol. There was no significant difference between the measured pressures in the apical area for the three protocols.

\section{Discussion}

Increasing efficacy and simultaneously providing safety when irrigating root canals remains the main goal in endodontic therapy. LAI was shown to be an effective method of irrigant activation, which, by generating high fluid flow rates as a result of cavitation phenomena, results in effective removal of residual remnants of root canal contents and biofilm adhered to the root canal wall. ${ }^{31}$ During the production of increased pressure by LAI, which enhances cleaning efficiency, the risk of apical extrusion of irrigants remains. $^{27}$

This study aimed at measuring the distribution of pressures along the depth of a simulated root canal, for different parameters of LAI. Several methods for measurement of periapical pressures generated by different irrigating and activating techniques have been proposed in the literature so far. Usually, the measuring method is based on using a pressure sensor. $^{32,33}$ For example, in an LAI study, ${ }^{27}$ the pressure generated outside the apex was measured by using a digital positive pressure sensor. LAI generated similar or lower pressure outside the apical foramen compared with conventional syringe irrigation, agreeing with other apical extrusion studies, indicating that the extrusion during LAI is smaller than with standard syringe irrigation. ${ }^{6,22,34}$

As compared with the standard pressure sensor method, an advantage of the steady-state fluid level method used in this study is that it allows simultaneous pressure measure- ments at several closely spaced locations (Fig. 1b), with the number of locations being, in principle, limited only by the camera's measuring range. Although at a first glance the root canal model appears to be open at the measurement locations, there is a balance established between the outward and inward fluid flow rate when the measurement of $h_{i}$ is made at the steady state. Therefore, the measurement system can be considered to be closed, and representative for clinical situations.

The measurements were performed on a 3D-printed root canal model, which provided standardized working conditions. It should be noted that plastic material, in comparison to human dentine, has a different surface characteristic, which could have an impact on fluid dynamics. ${ }^{35}$

Our results demonstrate the importance of the design of the FT, which is determined by its size and shape. The measurements indicate that the generated pressure efficacy is higher for smaller FT diameters, and that conical FTs are less effective for generating pressures in comparison to cylindrical FTs. The highest efficacy was observed for the cylindrical flat-ended and tapered 400- $\mu$ s tips (Fig. 5), with no significant difference between the two FT types. For the larger FT diameter of $600 \mu \mathrm{s}$, the tapered FT was slightly more effective than flat-ended FT, in agreement with other reports. ${ }^{11,36}$ This is because tapered FTs generate spherically shaped bubbles where opto-dynamic energy conversion efficiency is optimal, ${ }^{36}$ whereas flat-ended FTs tend to generate more spheroid-shaped bubbles. This difference becomes less pronounced for smaller FT diameters in which bubbles become approximately spherical regardless of the FT ending.

No difference was observed between the SSP mode efficacies for the two lasers used in the study. However, a difference in the pressure efficacy was found between the SSP and AutoSWEEPS modes. Both LAI modes produced similar pressures at the apical level, whereas pressures obtained with the AutoSWEEPS at middle and coronal levels were found to be $20-40 \%$ higher in comparison to the SSP mode. The SWEEPS dual pulse modality is based on a finding that the collapse of a laser pulse-generated cavitation bubble can be accelerated by delivering a properly timed second laser pulse of the same energy. ${ }^{19,21,36}$ Since the proper timing depends on the cavitation bubble's oscillation time, which depends on the geometry of the access cavity, the AutoSWEEPS modality consists of automatic repetitive sweeping of the temporal separation between the SWEEPS pulse pairs back and forth between 250 and $650 \mu \mathrm{s}$, which covers all expected bubble oscillation times for all tooth types and access cavity sizes. This is intended to ensure that proper timing is achieved at least twice during each temporal separation sweep.

It is the accelerated collapse of the first bubble in the SWEEPS pulse pair that results in the enhanced shock wave emission and improved irrigation, whereas the role of the second bubble is mainly to amplify the effect of the first bubble. Also, during an endodontic procedure, the endodontist has to consider not to exceed the threshold for dentinal ablation that depends on the energy of individual laser pulses. $^{28}$ Accordingly, the laser energy for the SWEEPS modality $\left(E_{L}=10\right.$ and $\left.20 \mathrm{~mJ}\right)$ refers to the energy of the first pulse within the "SWEEPS" pulse pair.

To evaluate whether the penetration of irrigation solutions into dentinal tubules is related to the increased fluid pressures 
during LAI, additional pressure measurements were made by using the same LAI irrigation protocols as used in a recently published penetration study ${ }^{18,23}$ : (1) "PIPS" protocol (SSP mode, $E_{L}=20 \mathrm{~mJ}$, radial Sweeps600 FT) and (2) "SWEEEPS"' protocol (AutoSWEEPS mode, $E_{L}=10 \mathrm{~mJ}$, Conical Sapphire600 tip). The average of the pressures $P_{1}$ and $P_{2}$ was assigned to the average pressure in the apical area, the pressure $P_{3}$ to the average pressure in the middle area, and the average of pressures $P_{4}$ and $P_{5}$ to the average pressure in the coronal area. As shown in Fig. 7, a linear correlation was found between pressures measured in this study and published dentinal tubule penetration depths at apical, middle, and coronal root canal level with linear regression analysis giving $R^{2}=0.82$ and $p<0.01$.

It is also important to note that the results of this study are valid only for the Er:YAG laser wavelength $(\lambda=2.94 \mu \mathrm{m})$ and pulse durations and modalities used in the study. Namely, it is well known that the laser wavelength and pulse duration significantly influence the optodynamic (OD) phenomena during LAI. ${ }^{11,28,36}$ The OD energy-conversion efficiency, defined as the ratio between the bubble energy and the laser pulse energy, ${ }^{36}$ is smaller for wavelengths with longer optical penetration depth within the liquid, and longer pulse durations. Since the OD energy conversion efficiency of the erbium, chromium-doped yttrium, scandium, gallium and garnet (Er,Cr:YSGG) $(\lambda=2.73 \mu \mathrm{m})$ laser has been measured to be $\sim 4$ times lower in comparison to that of Er:YAG, ${ }^{11}$ it is expected that the pressures and dentinal tubule penetration depths during LAI performed with Er,Cr:YSGG laser will also be correspondingly smaller. However, pressure measurements with Er,Cr:YSGG will have to be made to confirm this conclusion.

There seems to be a lack of standardization regarding the reporting of the parameters, used in different studies, ${ }^{27,37,38}$ investigating the efficacy or safety of LAI. This could lead to misinterpretation or suboptimal performance of a given outcome of specific research on LAI. For example, in the penetration study just mentioned, ${ }^{18,23}$ the SSP and Auto-

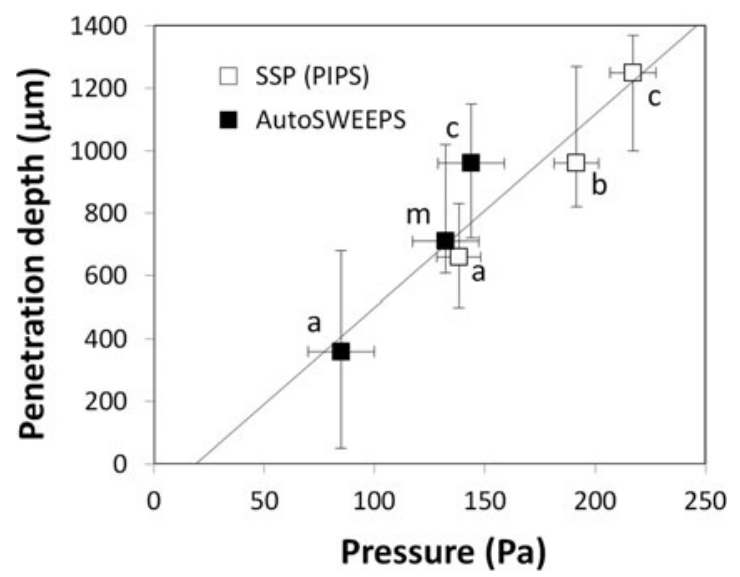

FIG. 7. Correlation between measured pressures and published dentinal tubule penetration depths ${ }^{18}$ at apical (a), middle $(\mathrm{m})$, and coronal (c) root canal level for two corresponding LAI modes: (1) SSP (Radial Sweeps600 FT; $E_{L}=20 \mathrm{~mJ}$ ) and (2) AutoSWEEPS (Conical Saphire600 FT; $\left.E_{L}=10 \mathrm{~mJ}\right) . R^{2}=0.82, p<0.01$. LAI, laser-activated irrigation.
SWEEPS modalities were not compared under identical conditions since different FTs were used for the two modalities. The AutoSWEEPS was, thus, performed by using the Conical Sapphire600 FT that is, according to the results of this study (Fig. 4), significantly inferior to the Radial Sweeps600 tip used with the "PIPS" protocol.

\section{Conclusions}

The generated pressures during LAI were observed to get progressively reduced from the coronal toward the apical third of the root canal. The pressure distribution within the root canal was found to be relatively well correlated with the published dentinal tubule penetration depths in coronal, middle, and apical areas. A strong dependence of the pressure generation on the FT design was observed. The pressure generation efficacy is higher for smaller FT diameters, with cylindrically shaped FTs being more effective in comparison to conically shaped FTs. Within the limitations of the study, our results indicate that when appropriate parameters are used, the AutoSWEEPS modality is more effective than standard SSP in generating pressures within the root canal, without increasing the risk of extrusion.

\section{Author Disclosure Statement}

N.L. and M.L. are also affiliated with Fotona d.o.o., Ljubljana, Slovenia.

\section{Funding Information}

This research was supported by the Ministry of Education, Science and Sport, Slovenia, under grants L3-7658, P20392, and Fotona d.o.o.

\section{References}

1. Peters OA. Current challenges and concepts in the preparation of root canal systems: a review. J Endod 2004;30: 559-567.

2. Peters OA, Schonenberger K, Laib A. Effects of four Ni-Ti preparation techniques on root canal geometry assessed by micro computed tomography. Int Endod J 2001;34:221-230.

3. Hulsmann M, Rummelin C, Schafers F. Root canal cleanliness after preparation with different endodontic handpieces and hand instruments: a comparative SEM investigation. J Endod 1997;23:301-306.

4. Ahmad M, Pitt Ford TR, Crum LA, Walton AJ. Ultrasonic debridement of root canals: acoustic cavitation and its relevance. J Endod 1988;14:486-493.

5. Bryce G, MacBeth N, Gulabivala K, Ng YL. The efficacy of supplementary sonic irrigation using the EndoActivator( $(\mathrm{R}))$ system determined by removal of a collagen film from an ex vivo model. Int Endod J 2018;51:489-497.

6. Charara K, Friedman S, Sherman A, et al. Assessment of apical extrusion during root canal irrigation with the Novel GentleWave System in a simulated apical environment. J Endod 2016;42:135-139.

7. Vyas N, Manmi K, Wang Q, et al. Which parameters affect biofilm removal with acoustic cavitation? A review. Ultrasound Med Biol 2019;45:1044-1055.

8. de Gregorio C, Estevez R, Cisneros R, Paranjpe A, Cohenca N. Efficacy of different irrigation and activation systems on the penetration of sodium hypochlorite into simulated lateral 
canals and up to working length: an in vitro study. J Endod 2010;36:1216-1221.

9. Peters OA, Bardsley S, Fong J, Pandher G, Divito E. Disinfection of root canals with photon-initiated photoacoustic streaming. J Endod 2011;37:1008-1012.

10. George R, Walsh LJ. Apical extrusion of root canal irrigants when using Er:YAG and Er,Cr:YSGG lasers with optical fibers: an in vitro dye study. J Endod 2008;34:706708.

11. Lukac N, Zadravec J, Gregorcic P, Lukac M, Jezersek M. Wavelength dependence of photon-induced photoacoustic streaming technique for root canal irrigation. J Biomed Opt 2016;21:75007.

12. Lukac N, Gregorcic, P, Jezersek, M. Optodynamic phenomena during laser-activated irrigation within root canals. Int J Thermophys 2016;37:66.

13. de Groot SD, Verhaagen B, Versluis M, Wu MK, Wesselink PR, van der Sluis LW. Laser-activated irrigation within root canals: cleaning efficacy and flow visualization. Int Endod J 2009;42:1077-1083.

14. Koch JD, Jaramillo DE, DiVito E, Peters OA. Irrigant flow during photon-induced photoacoustic streaming (PIPS) using particle image velocimetry (PIV). Clin Oral Investig 2016;20:381-386.

15. Arslan D, Kustarci A. Efficacy of photon-initiated photoacoustic streaming on apically extruded debris with different preparation systems in curved canals. Int Endod J 2018;51 e65-e72.

16. Olivi G, DiVito E. Photoacoustic endodontics using PIPS: experimental background and clinical protocol. LAHA 2012;1:22-25.

17. Matsumoto H, Yoshimine Y, Akamine A. Visualization of irrigant flow and cavitation induced by Er:YAG laser within a root canal model. J Endod 2011;37:839-843.

18. Galler KM, Grubmuller V, Schlichting R, et al. Penetration depth of irrigants into root dentine after sonic, ultrasonic and photoacoustic activation. Int Endod J 2019;52:12101217.

19. Lukac N, Jezersek M. Amplification of pressure waves in laser-assisted endodontics with synchronized delivery of Er:YAG laser pulses. Lasers Med Sci 2018;33:823-833.

20. Gregorcic P, Jamsek M, Lukac M, Mozina J, Jezersek M. Synchronized delivery of Er:YAG-laser-pulse energy during oscillations of vapor bubbles. LAHA 2014;17: 14-19.

21. Lukac N, Tasic Muc B, Jezersek M, Lukac M. Photoacoustic endodontics using the novel SWEEPS Er:YAG laser modality. LAHA 2017;2017:1-7.

22. Jezersek M, Jereb T, Lukac N, Tenyi A, Lukac M, Fidler A. Evaluation of apical extrusion during novel Er:YAG laser-activated irrigation modality. Photobiomodul Photomed Laser Surg 2019;37:544-550.

23. Akcay M, Arslan H, Mese M, Durmus N, Capar ID. Effect of photon-initiated photoacoustic streaming, passive ultrasonic, and sonic irrigation techniques on dentinal tubule penetration of irrigation solution: a confocal microscopic study. Clin Oral Investig 2017;21:2205-2212.

24. Boutsioukis C, Lambrianidis T, Kastrinakis E, Bekiaroglou $\mathrm{P}$. Measurement of pressure and flow rates during irrigation of a root canal ex vivo with three endodontic needles. Int Endod J 2007;40:504-513.

25. Shepherd PA, Eleazer PD, Clark SJ, Scheetz JP. Measurement of intraosseous pressures generated by the Wand, high-pressure periodontal ligament syringe, and the Stabident system. J Endod 2001;27:381-384.

26. Cai X, Wang XY, Santarcangelo F, et al. Effect of simulated intraosseous sinusoidal pressure on $\mathrm{NaOCl}$ extrusion. J Dent 2018;78:46-50.

27. Yao K, Satake K, Watanabe S, Ebihara A, Kobayashi C, Okiji T. Effect of laser energy and tip insertion depth on the pressure generated outside the apical foramen during Er: YAG laser-activated root canal irrigation. Photomed Laser Surg 2017;35:682-687.

28. Lukac M, Pustovrh G. Modeling photoacoustic efficiency during erbium laser endodontics. J Laser Health Acad 2013;2013.

29. Ostadfar A. Fluid mechanics and biofluid principles. In: Biofluid Mechanics. Elsevier, Inc., 2016; pp. 1-60. DOI: https//doi.org/10.1016/C2014-0-01583-3.

30. Yansheng L, Yancheng W, Xiuwu H, et al. HagenPoiseuille equation: a non-invasive tool for detecting renal pelvic pressure. Biomed Res 2017;28:2865-2869.

31. Al Shahrani M, DiVito E, Hughes CV, Nathanson D, Huang GT. Enhanced removal of Enterococcus faecalis biofilms in the root canal using sodium hypochlorite plus photon-induced photoacoustic streaming: an in vitro study. Photomed Laser Surg 2014;32:260-266.

32. Khan S, Niu LN, Eid AA, et al. Periapical pressures developed by nonbinding irrigation needles at various irrigation delivery rates. J Endod 2013;39:529-533.

33. Park E, Shen Y, Khakpour M, Haapasalo M. Apical pressure and extent of irrigant flow beyond the needle tip during positive-pressure irrigation in an in vitro root canal model. J Endod 2013;39:511-515.

34. Fukumoto Y, Kikuchi I, Yoshioka T, Kobayashi C, Suda $H$. An ex vivo evaluation of a new root canal irrigation technique with intracanal aspiration. Int Endod J 2006;39: 93-99.

35. Fukumoto Y. [Intracanal aspiration technique for root canal irrigation: evaluation of smear layer removal]. Kokubyo Gakkai Zasshi 2005;72:13-18.

36. Gregorcic P, Jezersek M, Mozina J. Optodynamic energyconversion efficiency during an Er:YAG-laser-pulse delivery into a liquid through different fiber-tip geometries. J Biomed Opt 2012;17:075006.

37. Yost RA, Bergeron BE, Kirkpatrick TC, et al. Evaluation of 4 different irrigating systems for apical extrusion of sodium hypochlorite. J Endod 2015;41:1530-1534.

38. Kustarci A, Er K. Efficacy of laser activated irrigation on apically extruded debris with different preparation systems. Photomed Laser Surg 2015;33:384-389.

Address correspondence to: Alě̌ Fidler, DMD, PhD Department of Endodontics University Clinical Centre Hrvatski trg 6 Ljubljana SI-1000 Slovenia

E-mail: ales.fidler@mf.uni-lj.si

Received: November 13, 2019. Accepted after revision: January 20, 2020. Published online: June 17, 2020. 